\title{
Study of the Byurakan-IRAS galaxy sample
}

\section{Areg M. Mickaelian and Gohar S. Harutyunyan}

Byurakan Astrophysical Observatory (BAO), Byurakan 0213, Aragatzotn province, Armenia email: aregmick@aras.am, goharutyunyan@gmail.com

\begin{abstract}
The Byurakan-IRAS Galaxy (BIG) sample (Mickaelian 1995) is the result of optical identifications of IRAS PSC sources at high-galactic latitudes using the First Byurakan Survey (FBS) low-dispersion spectra (Markarian et al. 1989). Among the 1577 objects 1178 galaxies have been identified. Most are dusty spiral galaxies and there are a number of ULIRGs among these objects. Our spectroscopic observations carried out with three telescopes (Byurakan Astrophysical Observatory 2.6m, Russian Special Astrophysical Observatory $6 \mathrm{~m}$ and Observatoire de Haute Provence 1.93m) (Mickaelian \& Sargsyan 2010) for 172 galaxies, as well as the SDSS DR8 spectra for 83 galaxies make up the list of 255 spectroscopically studied BIG objects. The classification to activity types for narrow-line emission galaxies has been carried out using the diagnostic diagrams by Veilleux \& Osterbrock (1987). All possible physical characteristics have been measured and/or calculated, including radial velocities and distances, angular and physical sizes, absolute magnitudes and luminosities (both optical and IR). IR luminosities and star-formation rates have been calculated from the IR fluxes (Duc et al. 1997).

Among the 172 observed galaxies, 102 starburst (HII) ones, 29 AGN (Sy or LINER), and 19 galaxies with composite spectrum have been revealed; spectra of 12 galaxies show emission features but without a possibility for more accurate classification, 9 galaxies are presented as galaxies where the star formation rate does not exceed normal one, and 1 galaxy is an absorption galaxy. Among the 83 objects having SDSS spectra, there are 55 HIIs, 8 Seyferts, 2 LINERs, 4 other AGN (without accurate classification), 6 composite spectrum objects and 8 other emissionline galaxies. In our spectroscopic sample we have 43 (17\%) AGN, 25 (10\%) composite spectrum objects, 157 (62\%) starbursts, 29 emission-line galaxies without a definite type, and 1 absorptionline galaxy. There are 3 Ultra-Luminous InfraRed Galaxies (ULIRG).

Various multiwavelength (MW) data have been retrieved for the full sample of 1178 objects from recent catalogs from X-ray to radio (ROSAT, GALEX, APM, MAPS, USNO, GSC, SDSS, 2MASS, WISE, IRAS, AKARI, NVSS, FIRST, etc.) to make a complete study of these galaxies possible. MW SEDs have been built, which have been matched to their optical classifications. Star-formation rates have been calculated to compare to their other physical characteristics, such as morphology, activity types, UV, optical, IR and radio luminosities, etc.
\end{abstract}

Keywords. surveys — infrared: galaxies — galaxies: active — galaxies: starburst

\section{References}

Duc, P. A., Mirabel, I. F., \& Maza, J. 1997, A\& AS, 124, 533

Markarian, B. E., Lipovetski, V. A., Stepanian, J. A., Erastova, L. K. \& Shapovalova, A. I. 1989 ComSAO, 62, 5

Mickaelian, A. M. 1995, Ap, 38, 625

Mickaelian, A. M. \& Sargsyan, L. A. 2010, Ap, 53, 483

Veilleux, S. \& Osterbrock, D. E. 1987, ApJS, 63, 295 